\title{
Relation of Plasma Selenium and Lipid Peroxidation End Products in Patients With Alzheimer's Disease
}

\author{
Z. CHMATALOVA ${ }^{1,3}$, M. VYHNALEK ${ }^{2,3}$, J. LACZO ${ }^{2,3}$, J. HORT ${ }^{2,3}$, R. POSPISILOVA ${ }^{1}$, \\ M. PECHOVA ${ }^{1}$, A. SKOUMALOVA ${ }^{1}$
}

${ }^{1}$ Department of Medical Chemistry and Clinical Biochemistry, Second Faculty of Medicine, Charles University and Motol University Hospital, Prague, Czech Republic, ${ }^{2}$ Memory Clinic, Department of Neurology, Second Faculty of Medicine, Charles University and Motol University Hospital, Prague, Czech Republic, ${ }^{3}$ International Clinical Research Center, St. Anne's University Hospital Brno, Brno, Czech Republic

Received January 12, 2017

Accepted April 18, 2017

On-line September 22, 2017

\section{Summary}

Increased oxidative stress in the brain during the course of Alzheimer's disease (AD) leads to an imbalance of antioxidants and formation of free radical reaction end-products which may be detected in blood as fluorescent lipofuscin-like pigments (LFPs). The aim of this study was to evaluate and compare LFPs with plasma selenium concentrations representing an integral part of the antioxidant system. Plasma samples from subjects with $A D$ dementia ( $A D D ; n=11$ ), mild cognitive impairment (MCI; $\mathrm{n}=17)$ and controls $(\mathrm{n}=12)$, were collected. The concentration of selenium was measured using atomic absorption spectroscopy. LFPs were analyzed by fluorescence spectroscopy and quantified for different fluorescent maxima and then correlated with plasma selenium. Lower levels of selenium were detected in $\mathrm{MCI}$ and ADD patients than in controls $(P=0.003$ and $P=0.049$, respectively). Additionally, higher fluorescence intensities of LFPs were observed in $\mathrm{MCI}$ patients than in controls in four fluorescence maxima and higher fluorescence intensities were also observed in MCI patients than in ADD patients in three fluorescence maxima, respectively. A negative correlation between selenium concentrations and LFPs fluorescence was observed in the three fluorescence maxima. This is the first study focused on correlation of plasma selenium with specific lipofuscin-like products of oxidative stress in plasma of patients with Alzheimer's disease and mild cognitive impairment.

\section{Key words}

Alzheimer's disease • Mild cognitive impairment • Lipofuscin-like pigments • Oxidative stress • Selenium

\section{Corresponding author}

Z. Chmátalová, Department of Medical Chemistry and Clinical Biochemistry, Second Faculty of Medicine, Charles University and Motol University Hospital, Plzeňská 130/221, 15006 Prague 5, Czech Republic. E-mail: zuzana.chmatalova@seznam.cz

\section{Introduction}

Oxidative stress is implicated in a pathophysiology of numerous neurodegenerative disorders including Alzheimer's disease (AD). AD is characterized by the accumulation of amyloid plaques, the formation of neurofibrillary tangles and the loss of synapses followed by atrophy (Braak and Braak 1991) that manifests clinically as a progressive cognitive decline. The dementia stage of AD (ADD) is preceded by mild cognitive impairment (MCI), a prodromal stage of $\mathrm{AD}$, in which brain damage is not severe enough to compromise self-sufficiency. The MCI patients with objective memory impairment are labelled as amnestic MCI (aMCI), their rate of progression to ADD is high: $15 \%$ of them convert to ADD every year (Petersen 2004).

Oxidative stress is considered to be one of important pathogenic factor in the development and progression of AD (Zhao and Zhao 2013). Nervous tissue is particularly vulnerable to the deleterious effects of free radicals for several reasons. The ATP used by neurons is 
produced primarily by oxidative phosphorylation, during which free radicals are generated. Moreover, the membranes of neurons are rich in polyunsaturated fatty acids, and the concentration of metal ions is high, which enables lipoperoxidation. In addition, the concentration of antioxidants in the brain is low in comparison to other tissues (Gilkun-Sherky et al. 2001). Finally, neurons are post-mitotic cells, in which oxidative damage products accumulate during the life span (Coyle and Puttfarcken 1993).

Oxidative stress markers are increased during all stages of the disease. It was shown that AD patients have increased levels of 4-hydroxynonenal and malondialdehyde in blood, which are markers of free radical-induced lipid peroxidation, as well as increased levels of F2-isoprostane and F4-neuroprostane in the brain tissue (Markesberry et al. 2005, Praticó et al. 1999). The above-mentioned markers of lipoperoxidation are general markers. More advanced and specialized end products of lipid free radical damage are represented by lipofuscin-like pigments (LFPs) (Skoumalova and Hort 2012). These pigments exhibit native fluorescence due to their cyclic structures and can be detected using fluorescence spectroscopy (Wilhelm and Herget 1999). Increased levels of LFP in the blood were previously found in ADD and MCI patients in comparison to controls (Skoumalova et al. 2011, Chmátalová et al. 2016).

All aerobic organisms have a very complex and effective antioxidant system, which protects molecules against oxidative damage. Selenium plays a major role in antioxidant defense via the redox regulation of key enzymes (Naziroǵlu and Yürekli 2013). Selenium is incorporated into proteins such as selenocysteine, which is a part of important antioxidant enzymes, particularly glutathione peroxidase and thioredoxin reductase. Moreover, selenium attenuates amyloid beta production and amyloid beta-induced neuronal death by reducing the activities of beta and gamma secretases (Gwon et al. 2010). Several studies of selenium plasma levels were provided in a recent years, but dissonant results of selenium assessment were obtained (Cardoso et al. 2014, Krishnan and Rani 2014).

In our previous study we found increased plasma levels of LFPs in patients with ADD and MCI (Chmátalová et al. 2016). The aim of this follow-up study was to compare plasma selenium levels in patients with aMCI, patients with ADD and to correlate it with fluorescence intensities of plasma LFPs. We wanted to evaluate whether lipoperoxidation end product overproduction in plasma, which might be used as a possible marker of neurodegeneration in $\mathrm{AD}$, is accompanied by selenium imbalance. Moreover, selenium measurement could be an additional referent analyte for higher robustness of LFPs measurement.

\section{Materials and Methods}

\section{Subjects}

All participants were recruited at the Memory Clinic at Motol University Hospital in Prague, Czech Republic, between 2012 and 2014. The group consisted of patients with mild ADD $(n=11)$, patients with aMCI $(n=17)$ and cognitively healthy older adults (controls, $\mathrm{n}=12$ ). All subjects underwent a complex diagnostic procedure including brain MRI, neuropsychological examination by neuropsychological battery, neurological examination. The diagnosis was made upon agreement between neurologist and neuropsychologist. Patients with clinically defined stroke, other neurological diseases (i.e. Parkinson disease, etc.), subjects with a history of diabetes, impaired glucose tolerance, hyperlipidemia, depression and significant vascular impairment on brain MRI ( $>2$ points on Fazekas scale) were not included (Fazekas et al. 1987).

The subjects with mild ADD met the National Institute on Aging - Alzheimer's Association criteria for probable AD without biomarker evidence (McKhan et al. 2011).

The subjects with aMCI met clinical criteria for aMCI, including memory complaints reported by a patient or a caregiver, evidence of memory dysfunction on neuropsychological testing, generally intact activities of daily living, and the absence of dementia (Petersen 2004). Memory impairment was established when the patient scored more than 1.5 standard deviations below the mean age- and education-adjusted norms on any memory test (Laczo et al. 2011).

The control group of cognitively healthy individuals was recruited from older adults attending the University of the Third Age at Charles University in Prague or from relatives of patients of the Memory Clinic at Motol University Hospital in Prague. Participants who met the DSM IV-TR criteria for dementia, Petersen's criteria for MCI (Petersen 2004) or who scored more than 1.5 SD below age- and education-adjusted norms on neuropsychological examination were not included. Characteristics of the groups are listed in Table 1. 
Table 1. Participants' characteristics of the tested groups.

\begin{tabular}{lcccc}
\hline & Men & Age range & Average age \pm SD & MMSE score \pm SD \\
\hline$A D D(n=11)$ & $7(64 \%)$ & $68-85$ & $74.27 \pm 5.14$ & $19.40 \pm 3.17$ \\
aMCI $(n=17)$ & $13(76 \%)$ & $56-88$ & $71.88 \pm 7.73$ & $25.14 \pm 3.25$ \\
Controls $(n=12)$ & $3(25 \%)$ & $69-85$ & $75.33 \pm 6.68$ & $28.40 \pm 1.71$ \\
\hline
\end{tabular}

MMSE score - Mini Mental State Exam score.

\section{Sample collection}

Five milliliters of blood from all subjects were collected into tubes coated with $\mathrm{K}_{3}$ EDTA. Plasma was obtained from fasting morning blood samples. All samples were centrifuged at $4,000 \mathrm{~g}$ for $5 \mathrm{~min}$ immediately after collection. Separated plasma was removed into a new tube and stored at $-80{ }^{\circ} \mathrm{C}$. The tubes were labelled with codes to ensure donor anonymity. All participants signed an informed consent before sample collection. This study was approved by the Ethics Committee of Motol University Hospital.

\section{Selenium assay}

The selenium assay was performed in the Department of Medical Chemistry and Clinical Biochemistry of Motol University Hospital in Prague, Czech Republic. The selenium concentration in the plasma was determined using atomic absorption spectrometry (atomic absorption spectrometer VARIAN SpectrAA 220 FS). According to the guidelines of Ministry of Health of the Czech Republic, the physiological range for selenium in plasma is 59.2-146.8 $\mu \mathrm{g} / 1$ (Statement of Ministry of Health of the Czech Republic).

\section{LFP assay}

LFP from the plasma was extracted into organic solvents using the method described by Goldstein and McDonagh, with modifications (Goldstein and McDonagh 1976). $2 \mathrm{ml}$ of plasma were added into the $6 \mathrm{ml}$ of mixture of organic solvents and LFPs were extracted for $1 \mathrm{~h}$. The chloroform phase was collected after centrifugation for fluorescence analysis using an AMINCO Bowman series 2 spectrophotometer. Fluorescence analyses of LFP in plasma were based on the measurement of synchronous fluorescence spectra. Synchronous spectra were measured for emission in the range of 250-500 nm with the fixed difference for excitation 25 and $50 \mathrm{~nm}$. The relative fluorescence intensity of different fluorescence maxima characterized by specific excitation and emission wavelengths were analyzed. The fluorescence maxima at $355 / 380 \mathrm{~nm}$ (excitation/emission), $350 / 400 \mathrm{~nm}$ and $360 / 410 \mathrm{~nm}$ were identified. The fluorescence intensity at $260 / 480 \mathrm{~nm}$ excitation/emission was used as a reference for the calculation of relative amounts of LFPs. The LFP measurement procedure and its principles were described in detail in our previous work (Chmátalová et al. 2016).

\section{Statistical analysis}

All statistical analyses were performed using the statistical programme SigmaPlot. To asses a normality of distribution Shapiro-Wilk test was used. An analysis of variance (ANOVA) with post hoc Holm-Sidak method was performed to evaluate differences between groups. Statistical significance was set at 2-tailed alpha of 0.05 . Pearson's correlation coefficients were used to estimate the associations between selenium concentrations and LFPs, cognitive measures and age. The results are reported as the mean values \pm standard deviations (SD).

\section{Results}

No statistically significant difference in the age of participants was observed between groups in this study.

\section{Selenium concentration}

The plasma selenium concentration was significantly higher in the control group than in the ADD $(\mathrm{P}=0.049)$ and aMCI $(\mathrm{P}=0.003)$ groups. Significant difference between selenium concentration in ADD and aMCI group was not observed. Mean selenium concentration in plasma was $76.07 \pm 18.45 \mu \mathrm{g} / 1$ in ADD group, $69.63 \pm 14.71 \mu \mathrm{g} / \mathrm{l}$ in aMCI and $90.72 \pm 17.56 \mu \mathrm{g} / \mathrm{l}$ in the control group. The comparison of selenium concentrations in patients and controls is shown in Figure 1 and Table 2. 


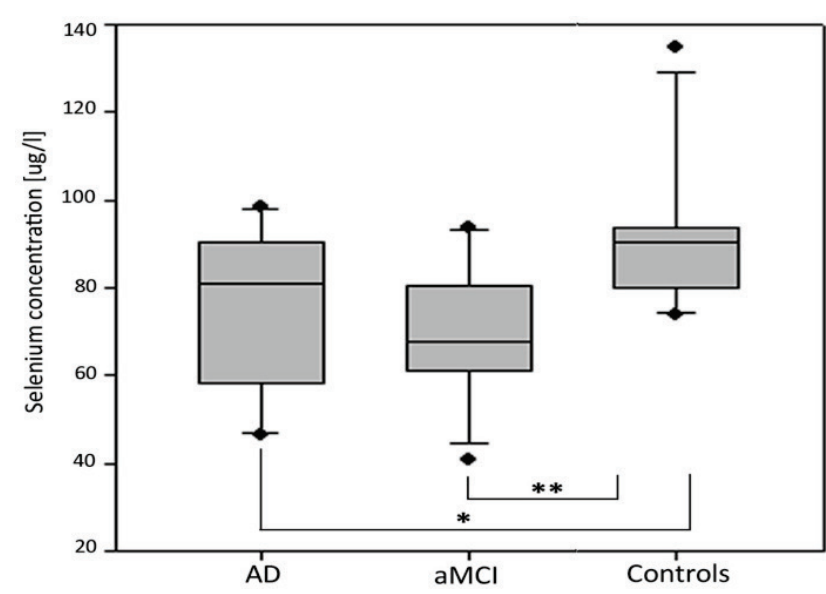

Fig. 1. Box plot representing comparison of selenium concentration in $\mathrm{AD}$ and $\mathrm{aMCI}$ patients and controls. Statistical significance: $* P<0.05$ and $* * P<0.01$.

Table 2. Selenium concentration ( \pm SD) in the plasma in $\mu \mathrm{g} / \mathrm{l}$ in ADD patients, aMCI patients and controls.

\begin{tabular}{lc}
\hline & Se concentration \pm SD $[\boldsymbol{\mu g} / \mathbf{l}]$ \\
\hline ADD & $76.07 \pm 18.45$ \\
aMCI & $69.63 \pm 14.71$ \\
Controls & $90.72 \pm 17.56$ \\
\hline
\end{tabular}

With respect to the physiological range for plasma selenium, all of our tested groups were in the recommended interval. However, some trends were observed. In the patient groups there were subjects with plasma selenium below the lower limit. In the ADD group 3 out of 11 and in the aMCI group 4 out of 17 subjects had selenium lower than $59.2 \mu \mathrm{g} / \mathrm{l}$. Given that age might influence selenium concentrations, a correlation analysis between selenium concentrations and age was performed. However, no significant correlation was found. Likewise, no correlation of selenium levels with Mini Mental State Exam (MMSE) scores was observed.

\section{LFP fluorescence intensity}

The fluorescence intensity in chloroform extracts from the plasma of ADD and aMCI patients and controls, which reflects the extent of oxidative damage, was measured. The results showed that fluorescence intensity is significantly higher in the aMCI patients compared to the controls in four specific fluorescence maxima $355 / 380 \mathrm{~nm}, 350 / 400 \mathrm{~nm}, 360 / 410 \mathrm{~nm}$ and $415 / 440 \mathrm{~nm}$ excitation/emission wavelength (355/380: $\mathrm{P}<0.001$; 350/400: $\mathrm{P}<0.001$; 360/410: $\mathrm{P}<0.001$; 415/440: $\mathrm{P}=0.003)$. A significant difference in fluorescence intensity was also observed between ADD and aMCI patients in terms of higher levels of LFP in aMCI compared to ADD. The significant difference was observed in three specific fluorescence maxima $355 / 380 \mathrm{~nm}, 350 / 400 \mathrm{~nm}$ and 415/440 nm (355/380: $\mathrm{P}=0.008 ; 350 / 400: \quad \mathrm{P}<0.001$; 415/440: $\mathrm{P}=0.010)$. The comparison of relative fluorescence intensities in specific fluorescence maxima between patients and control group is shown in Figure 2. Similar to our observations concerning selenium concentrations, no significant correlation between LFPs and MMSE and also no significant correlation between LFP levels and age of participants was observed.

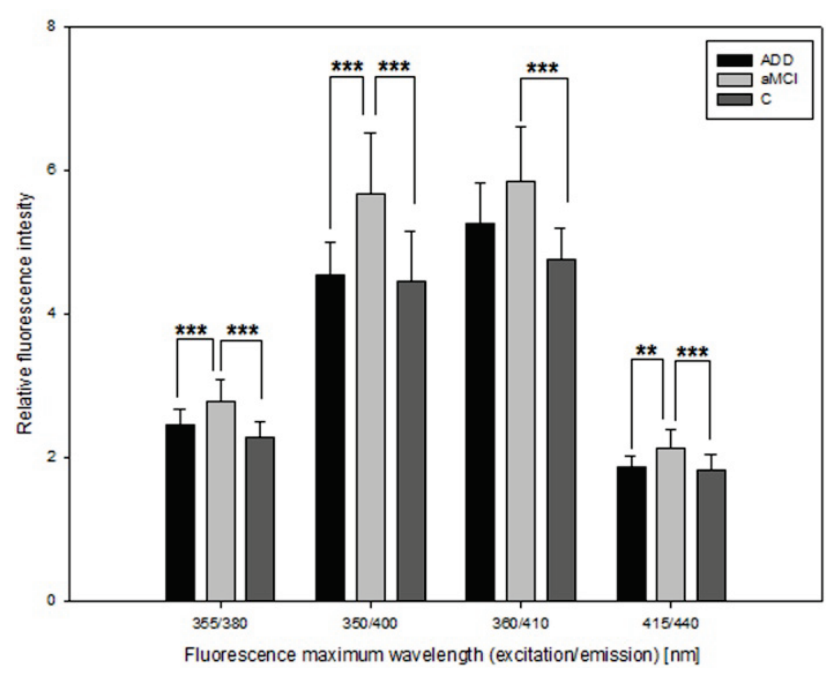

Fig. 2. The comparison of fluorescence intensities between the $\mathrm{ADD}$ and $\mathrm{aMCI}$ patient and control groups in four significant specific fluorescence maxima at $355 / 380 \mathrm{~nm}, 350 / 400 \mathrm{~nm}$, $360 / 410 \mathrm{~nm}$ and $415 / 440 \mathrm{~nm}$ (excitation/emission). Statistical significance: $* * \mathrm{P}<0.01$ and $* * * \mathrm{P}<0.01$.

\section{Association between selenium and LFP}

As selenium represents a part of the antioxidant defense system, its concentration was correlated with LFP levels, which are markers of oxidative stress. Pearson's correlation test showed that selenium concentrations correlated negatively with the amount of LFPs in plasma extracts at three fluorescence maxima: $355 / 380 \mathrm{~nm}(\mathrm{r}=-0.420 ; \mathrm{P}=0.012), 350 / 400 \mathrm{~nm}(\mathrm{r}=-0.390$; $\mathrm{P}=0.015)$ and $360 / 410 \mathrm{~nm}(\mathrm{r}=-0.398 ; \mathrm{P}=0.016)$. The correlation between selenium concentration and relative fluorescence intensity in specific fluorescent maxima is shown in Figure 3. 

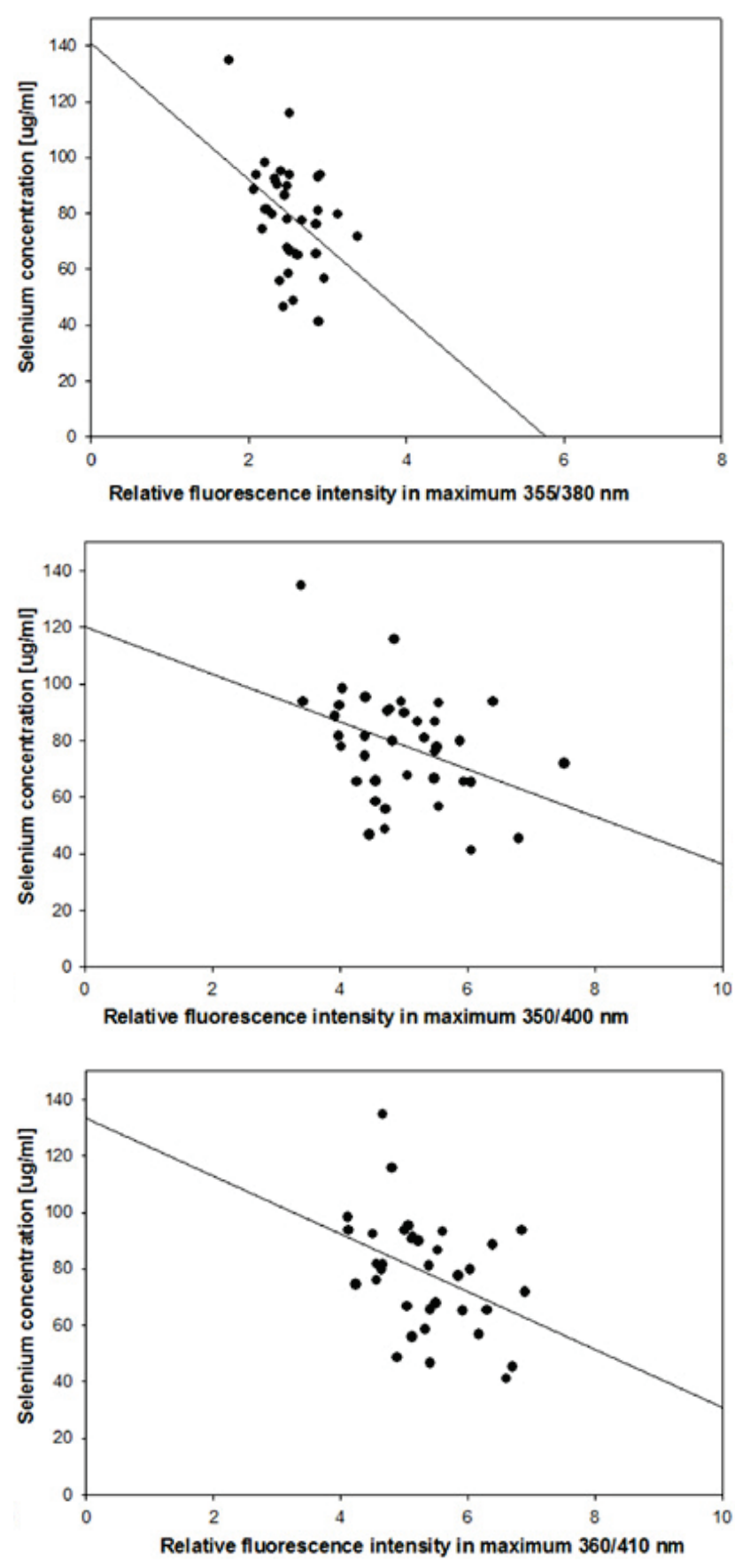

Fig. 3. Correlation graphs illustrating the correlation between selenium concentration and relative fluorescence intensity in three significant fluorescence maxima at $355 / 380 \mathrm{~nm}$ $(r=-0.420), \quad 350 / 400 \mathrm{~nm} \quad(r=-0.390)$ and $360 / 410 \mathrm{~nm}$ $(r=-0.398)$ (excitation/emission).

\section{Discussion}

Selenium plays an important role in the antioxidant defense and has been studied in many neurodegenerative diseases, including AD (Steinbrenner and Sies 2013). Some of these studies revealed lower selenium concentrations in plasma in $\mathrm{AD}$ patients compared to controls (Cardoso et al. 2014, GonzálesDomínguez et al. 2014), but couple of them did not proved significantly lower levels (Krishnan and Rani 2014, Koç et al. 2015).

In this study, we showed that although the mean selenium plasma concentrations in our tested groups were within the normal reported range (59.2-146.8 $\mu \mathrm{g} / \mathrm{l})$, the levels were significantly lower in patients with ADD and aMCI compared to controls. Moreover, many of patients in the ADD and aMCI groups had values below the normal range. Additionally, the recommended molar selenium concentration, which is required to achieve full glutathione peroxidase expression activity in the plasma, is in the range of 90.01-94.75 $\mu \mathrm{g} / 1$ (Thomson et al. 1993, Duffield et al. 1999). Consequently, our results showed that only the control group had sufficient selenium concentrations to support the maximal effective activity of the antioxidant enzyme glutathione peroxidase in the plasma.

Many studies demonstrated that increased oxidative stress leads to an increased production of free radical end products that arise from native biomolecules. But these commonly known markers of oxidative stress are not specific for particular pathologies. In contrast LFPs may represent such products with probable higher potential specificity for different diseases accompanied by oxidative stress and protein aggregation (Grune et al. 2004, Brunk and Terman 2002). Significantly higher levels of specific LFPs characterized by specific fluorescence intensities were detected in this study in plasma of ADD and aMCI patients compared to controls. These findings are consistent with the hypothesis that oxidative stress measured using non-specific markers is elevated during the course of $\mathrm{AD}$, which is even more pronounced during prodromal stages of AD (Nunomura et al. 2001, Ansari and Schew 2010). Further, these findings are consistent with results of our previous study showing higher levels of specific LFPs in erythrocytes and plasma among individuals with prodromal $\mathrm{AD}$ and ADD (Chmátalová et al. 2016).

The findings of the lower selenium levels and higher levels of specific LFPs in plasma among patients aMCI and ADD lead us to search for the associations between selenium levels and fluorescence intensities of LFPs. The correlation analysis revealed that there is a negative association between selenium concentrations and the amount of LFPs in plasma extracts. These results showed that the decreased selenium concentrations in patients with aMCI and ADD were related to higher 
detected fluorescence intensities of LFPs in plasma, which represent more pronounced oxidative stress damage. Consequently, decreased selenium concentrations in patients with aMCI and ADD might be the cause of attenuated glutathione peroxidase expression activity, which might lead to a higher rate of oxidative damage and increased formation of LFPs.

Despite relatively sufficient mean selenium levels, which were within a normal range in all three groups, we found significant differences in selenium concentration in patients with ADD and aMCI compared to controls. Moreover, significantly higher levels of oxidative stress markers in plasma among aMCI and ADD patients were observed. Regarding selenium concentration in the plasma, aMCI patients had the lowest levels, ADD patients were in the middle and the control group was found to have the highest selenium concentrations. The opposite findings were observed in terms of fluorescence intensities of LFPs, in aMCI most pronounced, in ADD in the middle and in controls less pronounced. These findings might be explained by increased demand for antioxidants in the course of AD development, in which oxidative stress is increased mainly in the early stages of the disease, including aMCI. Under conditions of increased oxidative stress, an increase in free radical defense is required. Biosynthesis of several antioxidants, such as glutathione peroxidase, thioredoxin reductase, selenoprotein $\mathrm{P}$ and methionine sulfoxide reductase $\mathrm{B}$, is dependent on the availability of selenium (Schweizer et al. 2004). That might explain the fact that patients with aMCI and ADD had significantly lower selenium in plasma compared to controls even though selenium food intake was sufficient in all tested groups.

The brain represents a high-priority organ that is supplied with selenium from other tissues if needed and that is the reason why the brain is the last organ to experience selenium deficiency (Cardoso et al. 2010). Consequently selenium concentration in plasma is rather short-term marker and selenium in blood and other organs might serve as a selenium pool for the brain in case of need in conditions of accelerated oxidative stress. This phenomenon might further explain our findings. The lowest effective concentration of selenium in plasma of aMCI patients correspond to the highest fluorescence intensities of LFP in plasma, which represent the marker of oxidative stress rate. In case of aMCI and ADD patients plasma selenium might be readily consumed for selenoprotein and other antioxidants synthesis not only in plasma, but preferentially in the brain to protect polyunsaturated fatty acids in the membranes of neurons. This might explain lower effective concentrations of selenium in plasma in patients and higher portion of free radical-damaged biomolecules in plasma. This hypothesis is in agreement with our previous work, in which we detected higher rate of lipoperoxidation of polyunsaturated fatty acids in membranes of erythrocytes in ADD and aMCI patients (Chmátalová et al. 2016). The lowest selenium concentrations in plasma of aMCI patients might be also explained by the fact that selenium attenuates the production of amyloid $\beta 42$ by reducing the activities of $\beta$ and $\gamma$ secretase in neuronal cell lines, which is more pronounced in the early stages including aMCI (Gwon et al. 2010).

Based on our results it might be hypothesized that a selenium diet supply as a therapeutic coadjutant approach might slow down the course of the disease or prevent the outcome. On the other hand, interventional studies with selenium supplementation were done with ambiguous results (Loef et al. 2011). This might be due to the fact that $\mathrm{AD}$ is multifactorial disease with long preclinical period and oxidative stress plays role in all of these stages.

Finally, no correlation between MMSE score and selenium or LFPs fluorescence intensities was found. Thus LFPs levels and selenium plasmatic concentrations seem to be independent markers of AD pathology regardless the stage of the disease.

In conclusion, our results revealed significantly lower plasma selenium concentrations in aMCI and ADD patients than in controls, which may be the consequence of the increased consumption of glutathione peroxidase and other selenium-dependent antioxidants in a course of $\mathrm{AD}$ development. These results were completed with measurement of specific end-products of oxidative damage, which proved significantly higher levels in aMCI and ADD patients than in controls.

\section{Conflict of Interest}

There is no conflict of interest.

\section{Acknowledgements}

This study was supported by the Grant Agency of Charles University (grant number 36216) and the 2nd Faculty of Medicine, Charles University, Prague; by the project no. LQ1605 from the National Program of Sustainability II (MEYS CR); by the European Regional Development 
Fund-Project FNUSA-ICRC (No. CZ.1.05/1.1.00/ 02.0123) and by the project ICRC-ERA-HumanBridge (No. 316345); European Social Fund within the project Young Talent Incubator II (Reg. No. CZ.1.07/2.3.00/
20.0117); Ministry of Health, Czech Republic conceptual development of research organization, Motol University Hospital 00064203, Prague, Czech Republic.

\section{References}

ANSARI MA, SCHEW SW: Oxidative stress in the progression of Alzheimer disease in the frontal cortex. J Neuropathol Exp Neurol 69: 155-167, 2010.

BRAAK H, BRAAK E: Neuropathological stageing of Alzheimer-related changes. Acta Neuropathol 82: 239-259, 1991.

BRUNK UT, TERMAN A: Lipofuscin: mechanisms of age-related accumulation and influence on cell function. Free Radic Biol Med 33: 611-619, 2002.

CARDOSO BR, ONG TP, JACOB-FILHO W, JALUUL O, FREITAS MI, COZZOLINO SMF: Nutritional status of selenium in Alzheimer's disease patients. Br J Nutr 103: 803-806, 2010.

CARDOSO RB, BANDEIRA SV, JACOB-FILHO W, COZZOLINO FSM: Selenium status in elderly: relation to cognitive decline. J Trace Elem Med Biol 28: 422-426, 2014.

CHMÁTALOVÁ Z, VYHNÁLEK M, LACZÓ J, HORT J, SKOUMALOVÁ A: Analysis of lipophilic fluorescent products in blood of Alzheimer's disease patients. J Cell Mol Med 20: 1367-1372, 2016.

COYLE JT, PUTTFARCKEN P: Oxidative stress, glutamate and neurodegenerative disorders. Science 262: 689-695, 1993.

DUFFIELD AJ, THOMSON CD, HILL KE, WILLIAMS S: An estimation of selenium requirements for New Zealanders. Am J Clin Nutr 70: 896-903, 1999.

FAZEKAS F, CHAWLUK JB, ALAVI A, HURTIG HI, ZIMMERMAN RA: MR signal abnormalities at $1.5 \mathrm{~T}$ in Alzheimer's dementia and normal aging. AJR Am J Roentgenol 149: 351-356, 1987.

GILGUN-SHERKI Y, MELAMED E, OFFEN D: Oxidative stress induced neurodegenerative diseases: the need for antioxidants that penetrate the blood brain barrier. Neuropharmacology 40: 959-975, 2001.

GOLDSTEIN BD, MCDONAGH EM: Spectrofluorescent detection of in vivo red cell lipid peroxidation in patients treated with diaminodiphenylsulphone. J Clin Invest 57: 1302-1307, 1976.

GONZÁLES-DOMÍNGUEZ R, GARCÍA-BARRERA T, GÓMEZ-ARIZA JL: Characterisation of metal profiles in serum during the progression of Alzheimer's disease. Metallomics 6: 292-300, 2014.

GRUNE T, JUNG T, MERKER K, DAVIES KJA: Decreased proteolysis caused by protein aggregates, inclusion bodies, plaques, lipofuscin, ceroid, and "aggresomes" during oxidative stress, aging and disease. Int J Biochem Cell Biol 36: 2519-2530, 2004.

GWON AR, PARK JS, PARK JH, BAIK SH, JEONG HY, HYUN DH, PARK KW, JO DG: Selenium attenuates A beta production and A beta-induced neuronal death. Neurosci Lett 469: 391-395, 2010.

KOÇ ER, ÍLHAN A, AYTÜRK Z, ACAR B, GÜRLER U, ALTUNTAŞ A, KARAPIRLİ M, BODUR AS: A comparison of hair and serum trace elements in patients with Alzheimer disease and healthy participants. Turk J Med Sci 45: 1034-1039, 2015.

KRISHNAN S, RANI P: Evaluation of selenium, redox status and their association with plasma amyloid/tau in Alzheimer's disease. Biol Trace Elem Res 158: 158-165, 2014.

LACZO J, ANDEL R, VLCEK K, MACOŠKA V, VYHNÁLEK M, TOLAR M, BOJAR M, HORT J: Spatial navigation and APOE in amnestic mild cognitive impairment. Neurodegener Dis 8: 169-177, 2011.

LOEF M, SCHRAUZER GN, WALACH H: Selenium and Alzheimer's disease: a systematic review. J Alzheimer's Dis 26: 81-104, 2011.

MARKESBERRY WR, KRYSCIO JR, LOVELL MA, MORROW JD: Lipid peroxidation is an early event in the brain in amnestic mild cognitive impairment. Ann Neurol 58: 730-735, 2005. 
MCKHANN GM, KNOPMAN DS, CHERTKOV H, HYMAN BT, JACK CR JR, KAWAS CH, KLUNK WE, KOROSHETZ WJ, MANLY JJ, MAYEUX R, MOHS RC, MORRIS JC, ROSSOR MN, SCHELTENS P, CARRILLO MC, THIES B, WEINTRAUB S, PHELPS CH: The diagnosis of dementia due to Alzheimer's disease: recommendations from the National Institute on Aging-Alzheimer's Association workgroups on diagnostic guidelines for Alzheimer's disease. Alzheimers Demen 7: 263-269, 2011.

NAZIROĞLU M, YÜREKLI VA: Effects of antiepileptic drugs on antioxidant and antioxidant molecular pathways: focus on trace elements. Cell Mol Neurobiol 33: 589-599, 2013.

NUNOMURA A, PERRY G, ALIEV G, HIRAI K, TAKEDA A, BALRAJ EK, JONES PK, GHANBARI H, WATAYA T, SHIMOHAMA S, CHIBA S, ATWOOD CS, PETERSEN RB, SMITH MA: Oxidative damage is the earliest event in Alzheimer disease. J Neuropathol Exp Neurol 60: 759-767, 2001.

PETERSEN RC: Mild cognitive impairment as a diagnostic entity. J Intern Med 256: 183-194, 2004.

PRATICÓ D, LEE VM, TROJANOWSKI JQ, ROKACH J, FITZGERALD GA: Increased F2-isoprostanes in Alzheimer's disease: evidence for enhanced lipid peroxidation in vivo. FASEB J 12: 1777-1783, 1999.

SCHWEIZER U, BRAÜER AU, KÖHRLE J, NITSCH R, SAVASKAN NE: Selenium and brain function: a poorly recognized liaison. Brain Res Rev 45: 164-178, 2004.

SKOUMALOVA A, HORT J: Blood markers of oxidative stress in Alzheimer's disease. J Cell Mol Med 16: 2291$2300,2012$.

SKOUMALOVA A, IVICA J, SANTOROVA P, TOPINKOVA E, WILHELM J: The lipid peroxidation products as possible markers of Alzheimer's disease in blood. Exp Gerontol 46: 38-42, 2011.

STATEMENT of Ministry of Health of Czech Republic: Selenium in plasma (in Czech). http://ciselniky.dasta.mzcr.cz/ hypertext/200620/hypertext/BOJVAAJ.htm. Accessed 31 ${ }^{\text {st }}$ May 2016.

STEINBRENNER H, SIES H: Selenium homeostasis and antioxidant selenoproteins in brain: implications for disorders in the central nervous system. Arch Biochem Biophys 536: 152-157, 2013.

THOMSON CD, ROBINSON MF, BUTLER JA, WHANGER PD: Long-term supplementation with selenate and selenomethionine: selenium and glutathione peroxidase (EC 1.11.1.19) in blood components of New Zealand women. Br J Nutr 69: 577-588, 1993.

WILHELM J, HERGET J: Hypoxia induces free radical damage to rat erythrocytes and spleen: analysis of the fluorescent end-products of lipid peroxidation. Int J Biochem Cell Biol 31: 671-681, 1999.

ZHAO Y, ZHAO B: Oxidative stress and the pathogenesis of Alzheimer's disease. Oxid Med Cell Longev 2013: 316523, 2013. 